\title{
A Node-Cooperative ARQ Scheme for Wireless Ad Hoc Networks
}

\author{
Mehrdad Dianati, Xinhua Ling, Kshirasagar Naik, Member, IEEE, and \\ Xuemin (Sherman) Shen, Senior Member, IEEE
}

\begin{abstract}
In this paper, the authors propose a nodecooperative automatic repeat request (ARQ) scheme for wireless ad hoc networks, which is suitable for mobile wireless channels with high and correlated frame-error profile. An analytical model based on a two-state Markovian process is proposed to describe the behavior of the proposed retransmission scheme and to obtain its throughput, average delay, and delay jitter. The results of Monte Carlo simulations are included to demonstrate the efficacy of the proposed scheme and to verify the accuracy of the analytical models. Results show that a cooperation among a small number of nodes can significantly improve the performance of the retransmission process in terms of throughput, average delay, and delay jitter by reducing the average duration of retransmission trials.
\end{abstract}

Index Terms-Automatic repeat request (ARQ), cooperative diversity, network performance, wireless ad hoc networks.

\section{INTRODUCTION}

C OMMUNICATIONS over wireless channels in an ad hoc network usually suffer from high frame-error rates due to the limited transmission power, the user mobility, and the lack of protection against interfering signals. Automatic repeat request (ARQ) schemes are de facto parts of wireless linklayer protocols to combat the high frame-error rate and to avoid expensive retransmissions of erroneous data blocks by the transport layer's error-control mechanism [2], [3]. Conventional ARQ schemes have been designed for wireline networks, where the frame errors are random. By random error, we mean that there is no correlation between frame-error probabilities for different frames. However, due to the inherent characteristics of the fading process in wireless channels, the frame errors appear in bursts rather than randomly. When the link between two communicating nodes is experiencing frame errors, there is a high probability that the bad channel condition will continue for a considerably long period. The duration of a bad channel condition or channel fading may be as long as the transmission time of multiple data frames. Conventional retransmission schemes are not very effective in such environments with

Manuscript received November 22, 2004; revised June 2, 2005 and August 2, 2005. This work was supported by a research grant from Bell University Laboratories (BUL) under the sponsorship of Bell Canada and the Natural Sciences and Engineering Research Council (NSERC) of Canada. The review of this paper was coordinated by Prof. Y.-B. Lin.

The authors are with the Center for Wireless Communications, Department of Electrical and Computer Engineering, University of Waterloo, Waterloo, ON N2L 3G1, Canada (e-mail: mdianati@bbcr.uwaterloo.ca; x2ling@bbcr. uwaterloo.ca; knaik@bbcr.uwaterloo.ca; xshen@bbcr.uwaterloo.ca).

Digital Object Identifier 10.1109/TVT.2005.863426 bursty frame errors and will cause significant degradation in the performance of a link-layer protocol [14].

Recently, spatial-diversity techniques in different forms of multiple transmit and receive antennas have been proposed to improve the quality of communication over wireless fading channels. Multiple input and multiple output (MIMO) communication systems [4], [5] and the corresponding channel coding techniques, such as space time coding (STC) [6], have been proposed to utilize space diversity in the next-generation wireless networks. However, the implementation of multiple antennas on small mobile devices is quite difficult due to the devicesize and cost constraints. An alternative form of space diversity can be achieved in a multiuser environment by allowing nodes to cooperate [1], [6], [7]. In cooperative communications, each node not only transmits and receives data for its own applications, but can also provide an alternative path for the other pairs of communicating nodes. In other words, each node acts as a relay node to facilitate better communications between other pairs of nodes at the link level.

The theoretical and implementation aspects of cooperative diversity in the physical layer have been areas of active interest among researchers [8]-[10]. However, to the best of our knowledge, the impacts of cooperative techniques on the upper layers of communication protocols have not been thoroughly studied so far.

In this paper, we propose a simple but effective ARQ scheme, namely node-cooperative stop and wait (NCSW) for wireless ad hoc networks. The proposed scheme is inspired by the idea of cooperative diversity in order to improve the performance of link-layer protocols. In the NCSW, nodes in the transmission range of each other form temporary cooperation groups. In conventional retransmission schemes, the neighbor nodes are oblivious to the ongoing transmission between a sender node and a receiver node. However, in the NCSW scheme, neighbor nodes that have enough resources and are willing to cooperate can assist the sender node in the retransmission process by retransmitting the same copy of the previous frame simultaneously. Intuitively, cooperation among individual nodes can increase the probability of a successful retransmission and mitigates negative impacts of the fading process. In order to investigate this intuition, we develop analytical models for the throughput, the average delay, and the delay jitter of the NCSW scheme by using a two-state Markov process for wireless fading channels. We present results of Monte Carlo simulations in order to demonstrate the better performance of the proposed scheme and to verify the accuracy of the analytical models. Both the analytical and the simulation results indicate that 


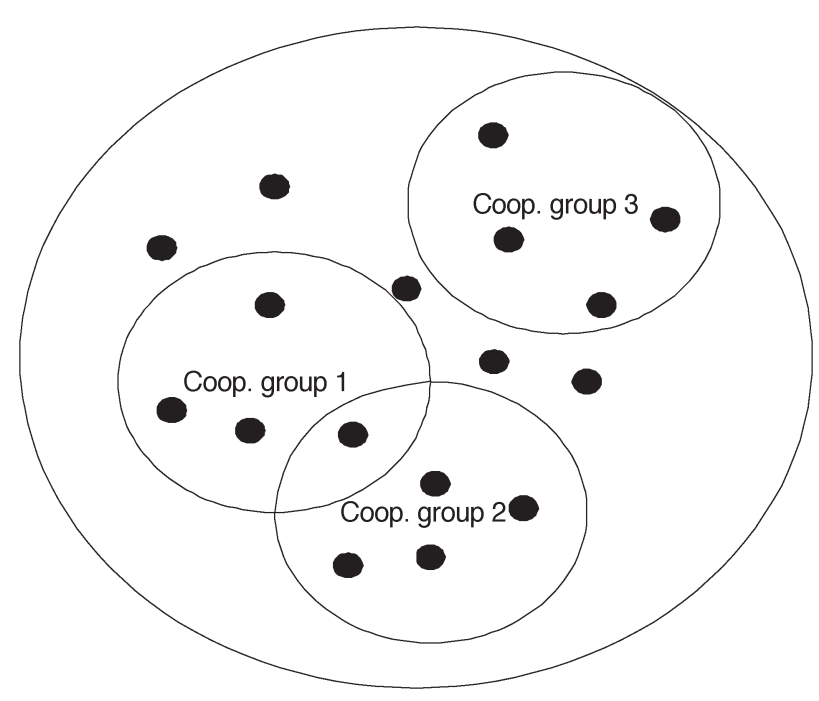

Fig. 1. Ad hoc wireless network model.

cooperation of a small number of nodes can produce significant performance gain, especially when the average quality of the channel between the sender and the receiver nodes is poor.

The rest of this paper is organized as follows. In Section II, the underlying assumptions and the system model are specified. The proposed ARQ scheme and the analytical models are presented in Section III. Simulation results are given in Section IV. Some concluding remarks and possible future research are given in Section $\mathrm{V}$.

\section{SySTEM ModeL}

We consider an ad hoc wireless network model as shown in Fig. 1. For the sake of generality, a group of autonomous nodes without any central control is considered. Although there is no fixed infrastructure, the model can generalize other types of wireless networks with infrastructures, such as cellular networks and wireless LANs (WLANs). In fact, a single node in this model can be a mobile device, a base station, or an access point. A cooperation group is a subset of nodes that can reach each other with a single hop. In other words, nodes in a cooperation group are in the radio coverage area of each other.

Cooperation groups may be set up during the connection setup stage (e.g., call setup in cellular networks or association in WLAN) or packet level handshaking. There is no need for extra signaling in order to form and manage cooperation groups. Cooperation groups are dynamic groups without tight membership requirement. Each neighbor node may join several cooperation groups depending on its position, capability, and willingness to cooperate. The overall structure of cooperation groups can be different in the transmission time of different packets. Thus, the mobility of nodes does not disrupt the operation. As shown in Fig. 2, each of those cooperation groups can be modeled as a single-hop wireless network. At any time instant, one sender node captures the shared media to send a burst of frames to its intended destination node. During that time period, the neighbor nodes in the group monitor the shared channel to assist the sender and the receiver nodes if an error happens. The whole process is transparent from a sender

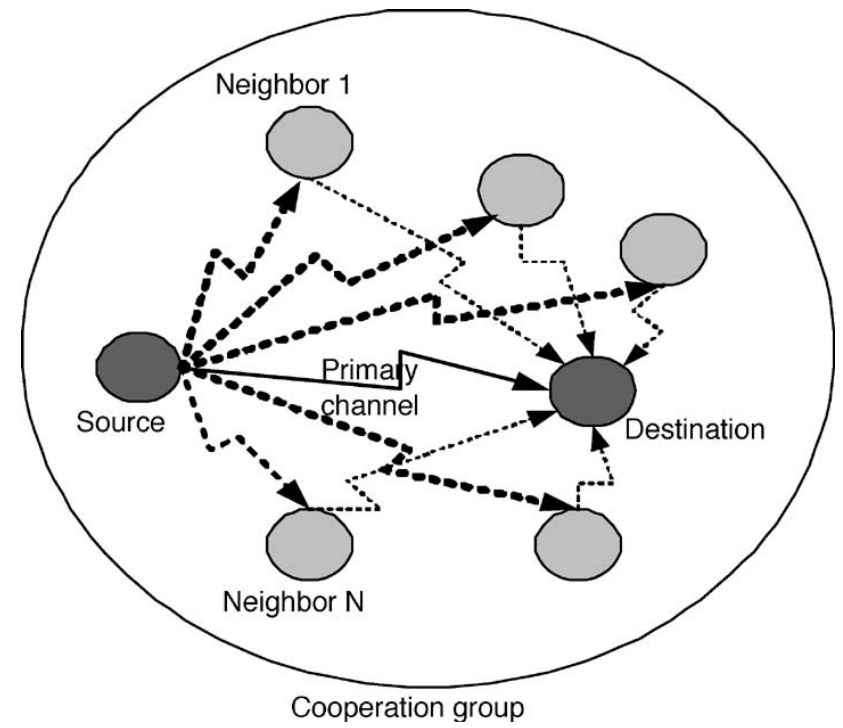

Fig. 2. Single cooperation group.

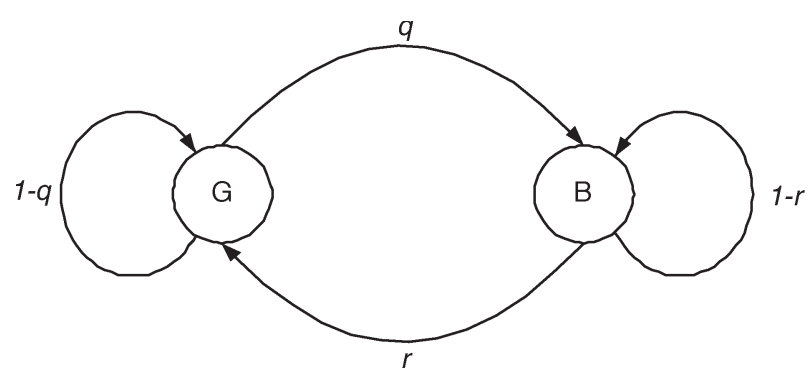

Fig. 3. Markov model for frame success/failure process over wireless fading channels.

node's point of view. Thus, a sender node performs its normal operation. A receiver node only needs to know the number and identity of the neighbor nodes during the current packet transmission time. Intuitively, node cooperation can improve the probability of a successful retransmission if there are extra resources available. Otherwise, all nodes perform their normal operations.

\section{A. Fading-Channel Model}

Variations of the wireless channel gains due to the destructive and constructive combination of multiple propagations with different path delays and center frequencies is known as a fading process. For flat fading channels, where the power spectrum density of the channel is constant over the whole frequency band of the information signal, a two-state Markov process as illustrated in Fig. 3 can adequately describe the process of a frame success or a failure [13]. The channel is deemed to be in the good state $(\mathrm{G})$ if a transmitted frame can be correctly decoded by the receiver; otherwise, when the channel is in the bad state (B), the transmitted frame cannot be decoded successfully and needs to be retransmitted by the sender.

If the channel is in state $G$ during the transmission time of the current frame, it will either stay in state $G$ with a probability $(1-q)$ or move to state B with a probability $q$ in the transmission time of the next frame. Similarly, starting 
from state $B$, the channel will either stay in state $B$ with a probability $(1-r)$ or move to state $\mathrm{G}$ with a probability $r$. Thus, the transition probability matrix for the two-state Markov process is

$$
\left[\begin{array}{cc}
1-q & q \\
r & 1-r
\end{array}\right]
$$

Let $\epsilon(k)$ be the frame success or failure process, where the discrete time index $k$ is the transmission of the $k$ th frame. Let $T_{\mathrm{f}}$ be the duration of a single frame. Assuming a nonadaptive channel coding rate and a quasi-static fading process (i.e., the fading level is constant for the entire frame duration), the transmitted frame $k$ cannot be decoded properly by the receiver if the channel power gain is below a certain threshold $\gamma$ at a time instant $k$, i.e.,

$$
\epsilon(k)= \begin{cases}\mathrm{B}, & \text { if }|\zeta(k)|^{2} \leq \gamma \\ \mathrm{G}, & \text { if }|\zeta(k)|^{2}>\gamma\end{cases}
$$

where $\zeta(k)$ represents the complex value of the fading envelope in the transmission time of frame $k$. For slow fading channels, the fading envelope can be considered constant for the entire frame duration, i.e., the fading envelope can be considered as a quasi-static process. For a quasi-static Rayleigh fading channel, i.e., $\zeta(k)$ has a Rayleigh probability distribution function (pdf), it has been shown in [14] that the transition parameters of the two-state Markov process in Fig. 3 can be given by

$$
\begin{aligned}
& r=\frac{Q(\theta, \rho \theta)-Q(\rho \theta, \theta)}{\mathrm{e}^{\gamma}-1} \\
& q=\frac{1-\mathrm{e}^{-\gamma}}{\mathrm{e}^{-\gamma}} r
\end{aligned}
$$

where $Q(\cdot, \cdot)$ is the Marcum $Q$ function

$$
\theta=\sqrt{\frac{2 \gamma}{1-\rho^{2}}}
$$

and

$$
\rho=J_{0}\left(2 \pi f_{m} T_{\mathrm{f}}\right)
$$

and $J_{0}(\cdot)$ is the zero-order Bessel function of the first kind. Similar Markovian models for the Rician and the Nakagami flat fading channels have been given in [15] and [16], respectively.

\section{B. Protocol Model}

In wireless ad hoc networks, the propagation delays among nodes are much smaller than the frame duration. For a link with a short propagation delay, a frame-by-frame acknowledgment mechanism such as the stop and wait (SW) transmission scheme is more efficient than the complex schemes such as the go back $N(\mathrm{GBN})$ and the selective repeat (SR) retransmission schemes. For example, the IEEE 802.11 compatible products adopt an SW-like retransmission scheme. Thus, in this paper, we adopt the SW as the core of the proposed ARQ scheme. For the SW ARQ scheme, the sender node does not transmit the next frame until the correct reception of the previous frame is confirmed by an explicit or implicit acknowledgment (ACK). We also assume that the feedback channels, used for transmitting ACK and negative acknowledgment (NAK), are error free. Thus, the ACK/NAK frames can be received immediately and correctly by all the nodes in a cooperation group. For the SW retransmission discipline with the aforementioned assumptions, given a two-state Markovian frame success/failure model for the wireless channel, the throughput of the SW ARQ scheme has been given in [17] as

$$
\eta_{\mathrm{SW}}=\frac{r}{r+q}
$$

where $q$ and $r$ are the parameters of the Markov model, as shown in Fig. 3.

\section{Node-CoOperative SW SCHEME}

Depending on the relative velocity of communicating nodes, the average duration of a channel fading can be as long as the transmission time of several frames. For example, for a slow fading channel with a node speed of $5 \mathrm{~km} / \mathrm{h}$ and a carrier frequency $f_{\mathrm{c}}=2.4 \mathrm{GHz}$, the average fading duration is about $60 \mathrm{~ms}$ [11]. For a typical frame length of $5 \mathrm{~ms}$, a naive retransmission scheme such as the conventional SW has to retransmit an erroneous frame for an average of 12 times. The situation may be even worse in high-rate systems with shorter frame durations.

Space diversity is a well-known technique in communication systems to combat fading in wireless channels by providing diverse and independent paths between a transmitter and a receiver. In our network model shown in Fig. 1, since the channel status between different pairs of nodes are independent of each other, node cooperation can be used to implement a variant of space diversity, which is known as a cooperative diversity [7]. Cooperation among those nodes that happen to be in the same radio coverage area of each other can significantly improve the performance of a retransmission scheme by reducing the number of unsuccessful retransmission trials.

Inspired by the cooperative diversity, we propose an NCSW ARQ scheme. The core concept can be explained using the model shown in Fig. 2, where a sender node is transmitting to a destination node and a typical neighbor node is listening to the ongoing communication. In the NCSW, the error-control procedures of the sender and the receiver nodes are the same as those of a conventional SW retransmission scheme in Fig. 4. However, the neighbor nodes in a cooperation group implement an additional functionality, as shown in Fig. 5. If a transmitted frame cannot be decoded successfully by the receiver, as part of its normal operation, the receiver node will send an NAK to the sender node asking for a retransmission of the erroneous frame, and the sender node will respond to the NAK by retransmitting the frame. In the conventional retransmission scheme, the neighbor nodes are oblivious to the retransmissions between the sender and the receiver nodes. However, in the NCSW scheme, all the other nodes in the cooperation group monitor the ongoing communications between the sender and the receiver nodes. The neighbor nodes decode and store a 
(a)

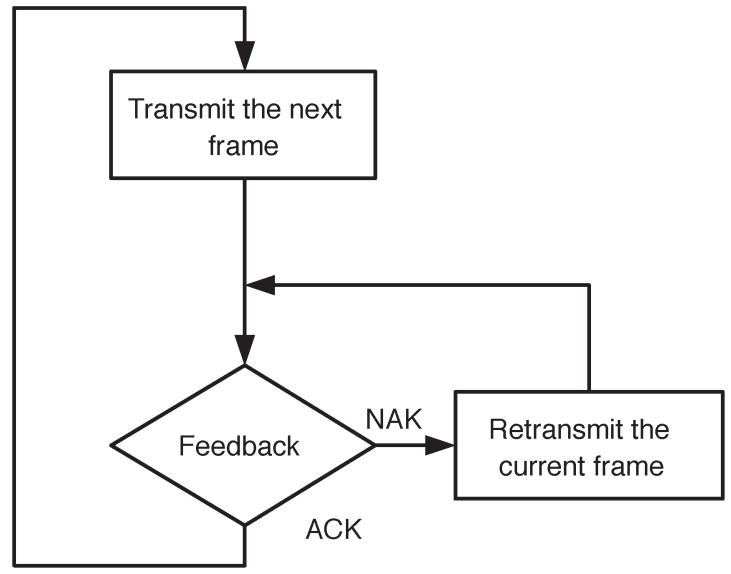

(b)

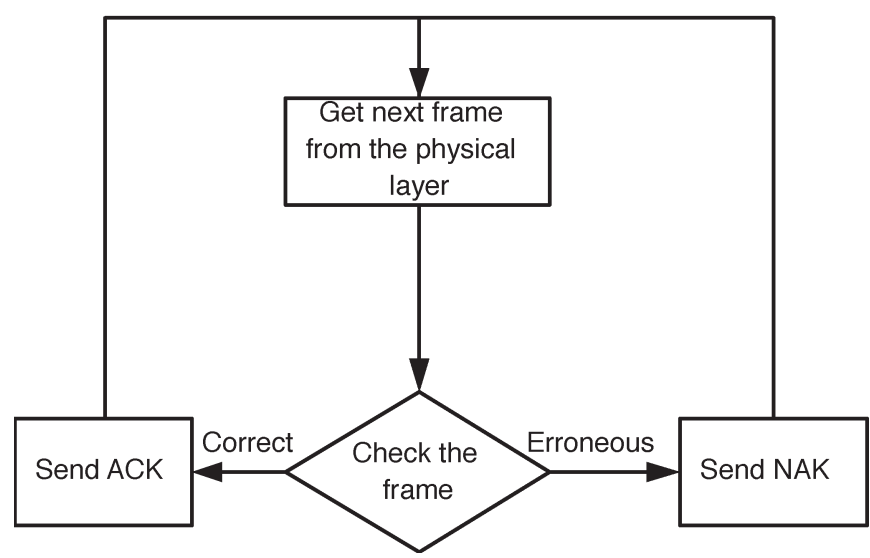

Fig. 4. SW ARQ (a) sender and (b) receiver.

copy of the last unacknowledged transmitted frame until the reception of a corresponding ACK. When a transmitted frame is acknowledged by the destination node, all the nodes in the cooperation group will drop their copy of the corresponding frame. However, when the neighbor nodes in a cooperation group receive an NAK from the destination node, they will cooperate with the sender in the retransmission process. Obviously, a node in a cooperation group can retransmit only if it has already received a correct copy of the requested frame. Even if a neighbor node has received a correct copy of the frame, cooperation is not mandatory, and each node may avoid cooperation for internal reasons or network considerations. This freedom of choice guarantees a backward compatibility of the NCSW protocol with the conventional SW protocol.

If proper coding and decoding schemes such as the distributed STC [12] are implemented in the physical layer, the probability of a successful retransmission will be significantly increased due to the diversity gain that can be achieved from the independent and diverse paths. Intuitively, if the channel coding scheme is capable of achieving a full diversity gain and all neighbor nodes have a correct copy of the frame, the probability of a successful retransmission for the NCSW scheme is $(1-$ $\left.\prod_{i}^{n} P_{\mathrm{e} i}\right)$, where $P_{\mathrm{e} i}$ is the probability of a frame error from node $i$ in the cooperation group to the destination node, and $n$ is the number of all the cooperating nodes plus the sender node.
Neighbor

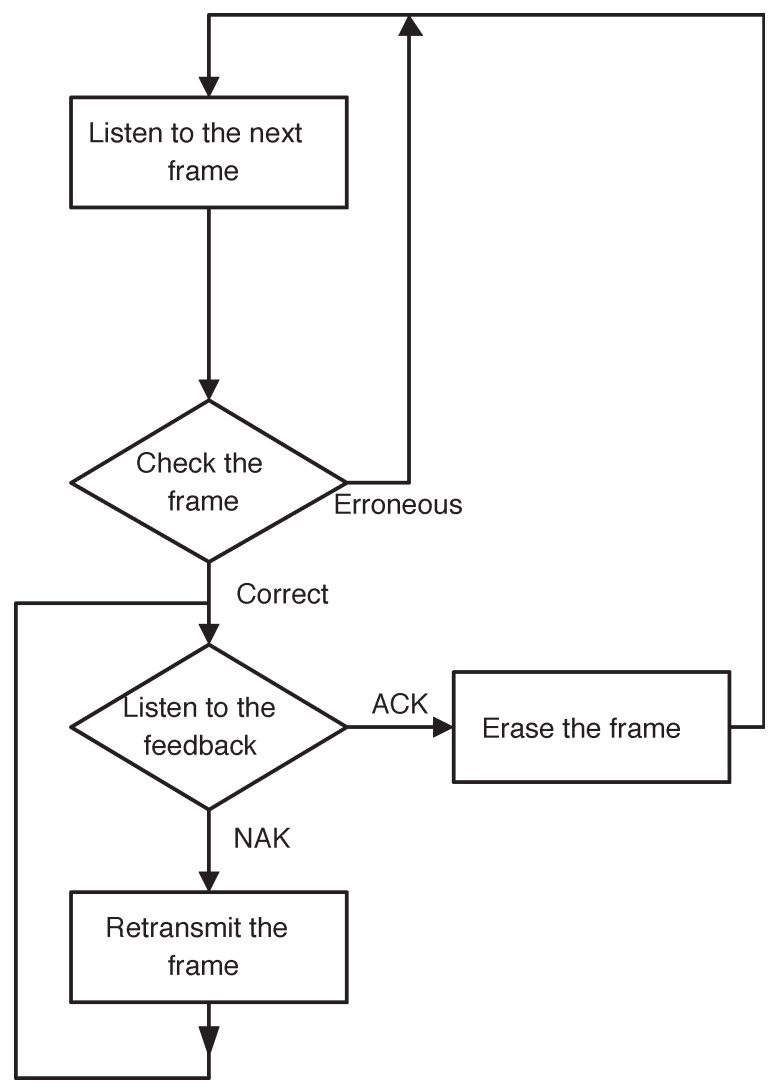

Fig. 5. Cooperation procedure in the NCSW ARQ.

\section{A. Protocol Analysis Model}

In this section, we develop an analytical model to describe the frame success/failure process for the proposed NCSW ARQ scheme. The model is a key component for analyzing useful performance metrics such as throughput, average delay, and delay jitter. We accomplish the analysis in three steps.

1) We derive a cooperation model for a pair of sender and receiver nodes with a single neighbor node.

2) We model the impacts of an arbitrary number of neighbor nodes in the cooperation group as an equivalent super neighbor node.

3) We combine the model for the super neighbor node from step 2 and the cooperation model from step 1 to obtain a model for the frame success/failure process for a pair of sender and receiver nodes and an arbitrary number of neighbor nodes.

In step 1 , we consider a sender/receiver pair of nodes with a single neighbor node as shown in Fig. 6. A two-state Markov model, as described in Section II, is used to specify a success or a failure of transmissions over a wireless fading channel. We use three distinct two-state Markov processes to model the primary channel from the sender node to the receiver node, the interim channel from the sender node to the neighbor node, and the relay channel from the neighbor node to the destination node. 


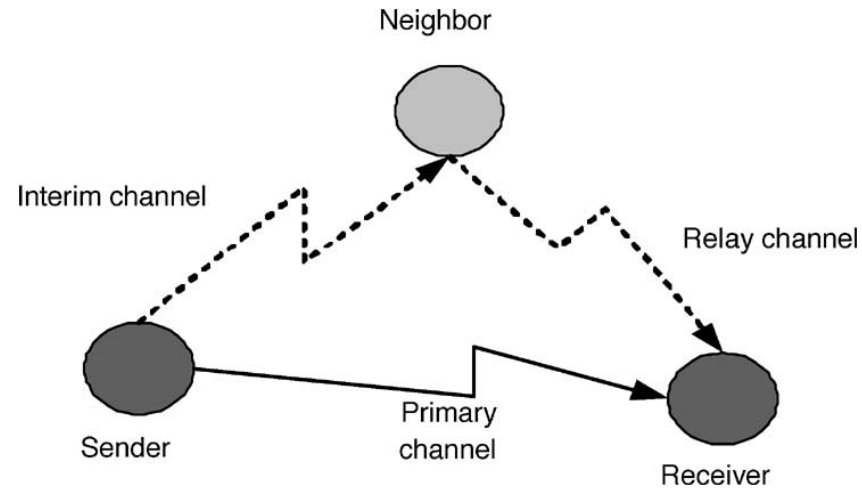

Fig. 6. System model for a single cooperative neighbor node.

The corresponding transition probability matrices are denoted as follows. For the primary channel

$$
\left[\begin{array}{cc}
1-q & q \\
r & 1-r
\end{array}\right]
$$

For the interim channel

$$
\left[\begin{array}{cc}
1-x & x \\
y & 1-y
\end{array}\right]
$$

For the relay channel

$$
\left[\begin{array}{cc}
1-a & a \\
b & 1-b
\end{array}\right]
$$

The corresponding transition probabilities are $(q, r),(x, y)$, and $(a, b)$, respectively. During the transmission time of frame $k$, the neighbor node is in state $\mathrm{B}$ if it is not able to cooperate in the possible retransmission of the $(k-1)$ th frame; otherwise, the neighbor node is considered to be in state G. Assuming that the neighbor node is always willing to cooperate, it will be in state $\mathrm{B}$ at time instant $k$ if frame $(k-1)$ cannot be decoded properly by that particular neighbor node, or the relay channel from that particular neighbor to the receiver node is in state $\mathrm{B}$ at the time instant $k$. With this argument, the status of the neighbor node at time instant $k$ can be formulated as follows:

$$
\mathrm{N}(k)= \begin{cases}\mathrm{G}, & \text { if } \mathrm{I}(k-1)=\mathrm{G} \text { and } \mathrm{R}(k)=\mathrm{G} \\ \mathrm{B}, & \text { otherwise }\end{cases}
$$

where $\mathrm{I}(k)$ and $\mathrm{R}(k)$ denote the states of the interim and relay channels, respectively.

A four-state Markov model, as shown in Fig. 7, can be used to represent the process of transition among different states of $\{\mathrm{I}(k-1), \mathrm{R}(k)\}$.

Let $\pi_{i}$ denote the probability of being in state $S_{i}$ in Fig. 7 . The following set of linear equations can be solved to obtain $\pi_{i}$ for $0 \leq i \leq 3$ :

$$
\begin{gathered}
{\left[\begin{array}{llll}
A_{00} & A_{10} & A_{20} & A_{30} \\
A_{01} & A_{11} & A_{21} & A_{31} \\
A_{02} & A_{12} & A_{22} & A_{32} \\
A_{03} & A_{13} & A_{23} & A_{33}
\end{array}\right]\left[\begin{array}{l}
\pi_{0} \\
\pi_{1} \\
\pi_{2} \\
\pi_{3}
\end{array}\right]=\left[\begin{array}{l}
\pi_{0} \\
\pi_{1} \\
\pi_{2} \\
\pi_{3}
\end{array}\right]} \\
\pi_{0}+\pi_{1}+\pi_{2}+\pi_{3}=1
\end{gathered}
$$

where $A_{i j}$ is the probability of transition from state $\mathrm{S}_{i}$ to state $\mathrm{S}_{j}$. As given in (4), $\mathrm{N}(k)$ can be modeled by another two-state Markov process with transition parameters of $(u, v)$, where $u$ and $v$ are defined as follows:

$$
\begin{aligned}
& u \triangleq P\{\mathrm{~N}(k)=\mathrm{B} \mid \mathrm{N}(k-1)=\mathrm{G}\} \\
& v \triangleq P\{\mathrm{~N}(k)=\mathrm{G} \mid \mathrm{N}(k-1)=\mathrm{B}\} .
\end{aligned}
$$

From the Markov process shown in Fig. 7, it can be seen that

$$
u=1-(1-x)(1-a)
$$

We can rewrite $v$ in (6) in a slightly different form as

$$
v=\frac{P\{\mathrm{~N}(k-1)=\mathrm{B} \mid \mathrm{N}(k)=\mathrm{G}\} P\{\mathrm{~N}(k)=\mathrm{G}\}}{P\{\mathrm{~N}(k-1)=\mathrm{B}\}} .
$$

The solution of (5) can be combined with (8) to obtain $v$ as

$$
v=\frac{u \pi_{0}}{1-\pi_{0}}
$$

The Markov process specified by (7) and (9) characterizes the status of a single neighbor node. In step 2, we propose an iterative approach to reduce a cooperation group with multiple neighbor nodes to an equivalent cooperation group with only one super neighbor node. Let $M \geq 2$ be the total number of neighbor nodes. In the first iteration, we combine neighbor nodes 1 and 2 into one equivalent node. After that, the resulting equivalent node is combined with node 3 , and so on, until all the $M$ neighbor nodes are combined together to form a single super neighbor node.

Let $\mathrm{N}^{(1)}(k)$ and $\mathrm{N}^{(2)}(k)$ be the states of neighbor nodes 1 and 2 at time instant $k$, respectively. Since a retransmission will succeed if at least one of the neighbor nodes or the sender node can successfully deliver the frame to the receiver node, the combined cooperative node model for nodes 1 and 2, denoted by $\mathrm{N}^{(1,2)}(k)$, can be represented by

$$
\mathrm{N}^{(1,2)}(k)= \begin{cases}\mathrm{G}, & \text { if } \mathrm{N}^{(1)}(k)=\mathrm{G} \text { or } \mathrm{N}^{(2)}(k)=\mathrm{G} \\ \mathrm{B}, & \text { otherwise. }\end{cases}
$$

The discrete random process $\mathrm{N}^{(1,2)}(k)$ as specified by (10) can be modeled by a two-state Markov process, where its parameters are defined by

$$
\begin{aligned}
& u^{(1,2)} \triangleq P\left\{\mathrm{~N}^{(1,2)}(k)=\mathrm{B} \mid \mathrm{N}^{(1,2)}(k-1)=\mathrm{G}\right\} \\
& v^{(1,2)} \triangleq P\left\{\mathrm{~N}^{(1,2)}(k)=\mathrm{G} \mid \mathrm{N}^{(1,2)}(k-1)=\mathrm{B}\right\} .
\end{aligned}
$$

Let $\left(u_{1}, v_{1}\right)$ and $\left(u_{2}, v_{2}\right)$ be the corresponding Markov parameters of $\mathrm{N}^{(1)}(k)$ and $\mathrm{N}^{(2)}(k)$, respectively. As explained in step $1,\left(u_{1}, v_{1}\right)$ and $\left(u_{2}, v_{2}\right)$ can be computed by (7) and (9) for nodes 1 and 2 , respectively. The status of $\left\{\mathrm{N}^{(1)}(k), \mathrm{N}^{(2)}(k)\right\}$ can be described by another four-state Markov process, as shown in Fig. 8.

From Fig. 8 and (11), it can be easily seen that

$$
v^{(1,2)}=1-\left(1-v_{1}\right)\left(1-v_{2}\right) .
$$




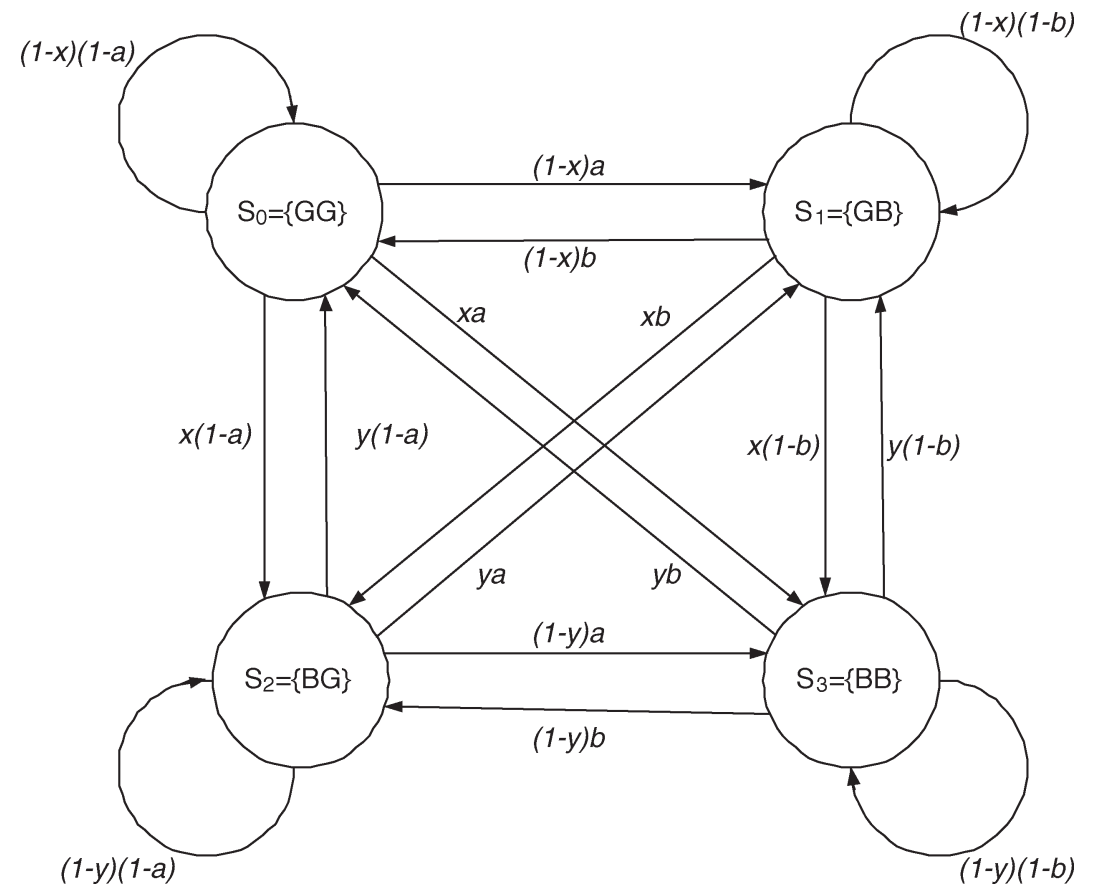

Fig. 7. State transition model for $\{\mathrm{I}(k-1), \mathrm{R}(k)\}$.

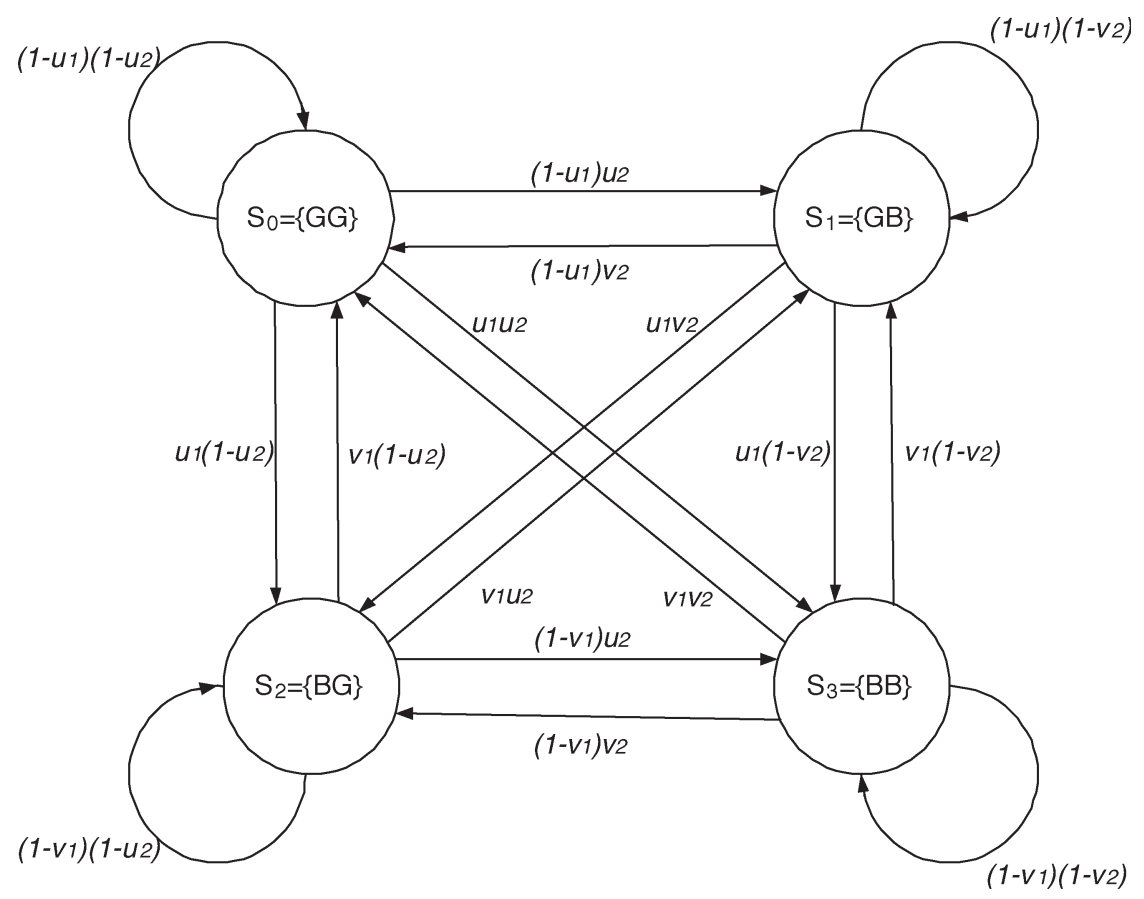

Fig. 8. State transition model for $\left\{\mathrm{N}^{(1)}(k), \mathrm{N}^{(2)}(k)\right\}$.

Using (12), we can obtain $u^{(1,2)}$ as

$$
\begin{aligned}
u^{(1,2)}= & P\left\{\mathrm{~N}^{(1,2)}(k-1)=\mathrm{G} \mid \mathrm{N}^{(1,2)}(k)=\mathrm{B}\right\} \\
& \cdot \frac{P\left\{\mathrm{~N}^{(1,2)}(k)=\mathrm{B}\right\}}{P\left\{\mathrm{~N}^{(1,2)}(k-1)=\mathrm{G}\right\}} \\
= & \frac{v^{(1,2)} \pi_{3}}{1-\pi_{3}}
\end{aligned}
$$

where $\left[\begin{array}{llll}\pi_{0} & \pi_{1} & \pi_{2} & \pi_{3}\end{array}\right]$ can be obtained by solving (5) in Fig. 8.

In the next iteration, the two-state Markov model specified by $\left(u^{(1,2)}, v^{(1,2)}\right)$ is combined with the two-state Markov model of neighbor node 3 , which is specified by $\left(u_{3}, v_{3}\right)$. The combination process follows the same steps that we used to combine neighbor nodes 1 and 2 . This process is repeated until all the neighbor nodes in the cooperation group are considered. Finally, we obtain a two-state Markov model for the super neighbor node, which includes the impacts of all neighbor 


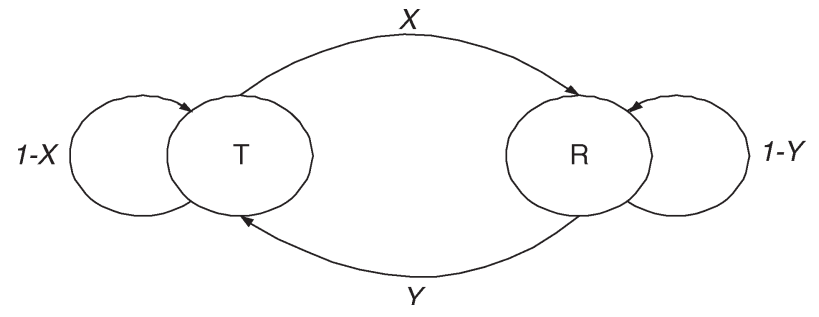

Fig. 9. Markov model for the NCSW ARQ.

nodes. We denote the status of the super neighbor node by $\mathrm{N}^{(1, \ldots, M)}(k)$ and use the following notations for its transition probabilities:

$$
\begin{aligned}
U & =u^{(1, \ldots, M)} \\
& \triangleq P\left\{\mathrm{~N}^{(1, \ldots, M)}(k)=\mathrm{B} \mid \mathrm{N}^{(1, \ldots, M)}(k-1)=\mathrm{G}\right\} \\
V & =v^{(1, \ldots, M)} \\
& \triangleq P\left\{\mathrm{~N}^{(1, \ldots, M)}(k)=\mathrm{G} \mid \mathrm{N}^{(1, \ldots, M)}(k-1)=\mathrm{B}\right\} .
\end{aligned}
$$

In step 3, we model the transmission and retransmission processes of the NCSW ARQ scheme with one sender node, one receiver node, and one super neighbor node. Let $\mathrm{O}(k)$ denote the state of the NCSW protocol at time instant $k . \mathrm{O}(k)$ is either in transmission (T) state or retransmission (R) state according to the two-state Markov model shown in Fig. 9.

The parameters of this Markov model are defined as

$$
\begin{aligned}
& X \triangleq P\{\mathrm{O}(k)=\mathrm{R} \mid \mathrm{O}(k-1)=\mathrm{T}\} \\
& Y \triangleq P\{\mathrm{O}(k)=\mathrm{T} \mid \mathrm{O}(k-1)=\mathrm{R}\} .
\end{aligned}
$$

In state $\mathrm{T}$, the sender transmits a new frame. However, in state $\mathrm{R}$, all nodes in the cooperation group retransmit the previously failed frame. Let $\operatorname{PC}(k)$ represent the state of the primary channel at time instant $k ; \mathrm{O}(k)$ will transit between the $\mathrm{T}$ and $\mathrm{R}$ states according to the logic given by Table I. This table specifies the transition logic for an eight-state Markov model, as shown in Fig. 10. The corresponding transition probability matrix $\mathbf{B}(8 \times 8)$ is given by

$$
\mathbf{B}=\left[\begin{array}{cccccccc}
0 & 0 & 0 & 0 & \bar{r} \bar{V} & \bar{r} V & r \bar{V} & r V \\
0 & 0 & 0 & 0 & \bar{r} U & \bar{r} \bar{U} & r U & r \bar{U} \\
q \bar{V} & q V & \bar{q} \bar{V} & \bar{q} V & 0 & 0 & 0 & 0 \\
q U & q \bar{V} & \bar{q} U & \bar{q} \bar{U} & 0 & 0 & 0 & 0 \\
0 & 0 & 0 & 0 & \bar{r} \bar{V} & \bar{r} V & r \bar{V} & r V \\
\bar{r} U & \bar{r} \bar{U} & r U & r \bar{U} & 0 & 0 & 0 & 0 \\
q \bar{V} & q V & \bar{q} \bar{V} & \bar{q} V & 0 & 0 & 0 & 0 \\
q U & q \bar{U} & \bar{q} U & \bar{q} \bar{U} & 0 & 0 & 0 & 0
\end{array}\right]
$$

where $q$ and $r$ are parameters of the Markov model for the primary channel, and $\overline{(\cdot)}=1-(\cdot)$. Let $\mathbf{P}=\left[p_{\mathrm{S}_{0}}, \ldots, p_{\mathrm{S}_{7}}\right]$ denote the steady-state-probability vector, where $p_{\mathrm{S}_{i}}$ is the steady-state
TABLE I

STATE TRANSITION LOGIC FOR O $(k)$

\begin{tabular}{c|c}
\hline$\left\{(k-1), P C(k-1), N^{(1, \ldots, M)}(k-1)\right\}$ & $O(k)$ \\
\hline$S_{0}:\{\mathrm{T}, \mathrm{G}, \mathrm{G}\}$ & $\mathrm{T}$ \\
$S_{1}:\{\mathrm{T}, \mathrm{G}, \mathrm{B}\}$ & $\mathrm{T}$ \\
$S_{2}:\{\mathrm{T}, \mathrm{B}, \mathrm{G}\}$ & $\mathrm{R}$ \\
$S_{3}:\{\mathrm{T}, \mathrm{B}, \mathrm{B}\}$ & $\mathrm{R}$ \\
$S_{4}:\{\mathrm{R}, \mathrm{G}, \mathrm{G}\}$ & $\mathrm{T}$ \\
$S_{5}:\{\mathrm{R}, \mathrm{G}, \mathrm{B}\}$ & $\mathrm{T}$ \\
$S_{6}:\{\mathrm{R}, \mathrm{B}, \mathrm{G}\}$ & $\mathrm{T}$ \\
$S_{7}:\{\mathrm{R}, \mathrm{B}, \mathrm{B}\}$ & $\mathrm{R}$ \\
\hline
\end{tabular}

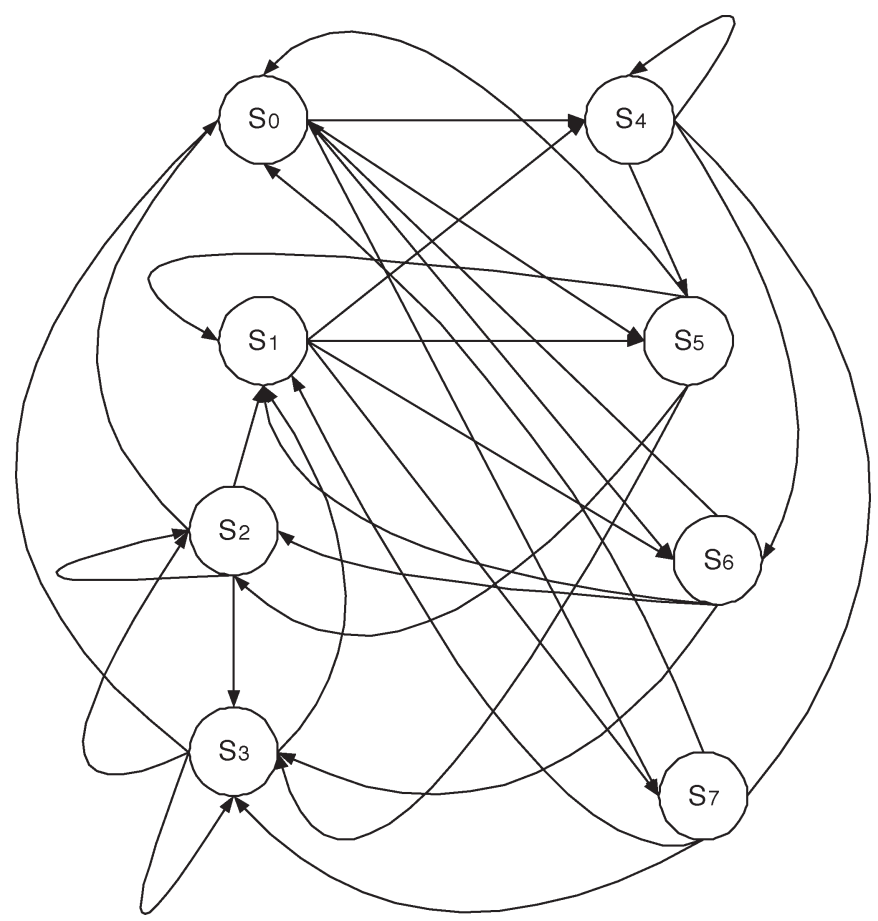

Fig. 10. Markov model for $\left\{\mathrm{O}(k-1), \mathrm{PC}(k-1), \mathrm{N}^{(1, \ldots, M)}(k-1)\right\}$.

probability of being in state $S_{i}$ in Fig. 10. This vector can be obtained by solving a set of linear equations given by

$$
\begin{aligned}
\mathbf{P} \cdot \mathbf{B} & =\mathbf{P} \\
p_{\mathrm{S}_{0}}+\ldots+p_{\mathrm{S}_{7}} & =1 .
\end{aligned}
$$

Having $p_{\mathrm{S}_{i}}$, for $i=0, \ldots, 7$, and Table $\mathrm{I}$, the parameters of the two-state Markov model for the NCSW protocol can be obtained by

$$
\begin{aligned}
X & =\frac{p_{\mathrm{S}_{2}}+p_{\mathrm{S}_{3}}}{p_{\mathrm{S}_{0}}+p_{\mathrm{S}_{1}}+p_{\mathrm{S}_{2}}+p_{\mathrm{S}_{3}}} \\
Y & =\frac{p_{\mathrm{S}_{4}}+p_{\mathrm{S}_{5}}+p_{\mathrm{S}_{6}}}{p_{\mathrm{S}_{4}}+p_{\mathrm{S}_{5}}+p_{\mathrm{S}_{6}}+p_{\mathrm{S}_{7}}} .
\end{aligned}
$$

Equation (18) completes the modeling of a frame success/ failure process as a two-state Markov model. Given this model 


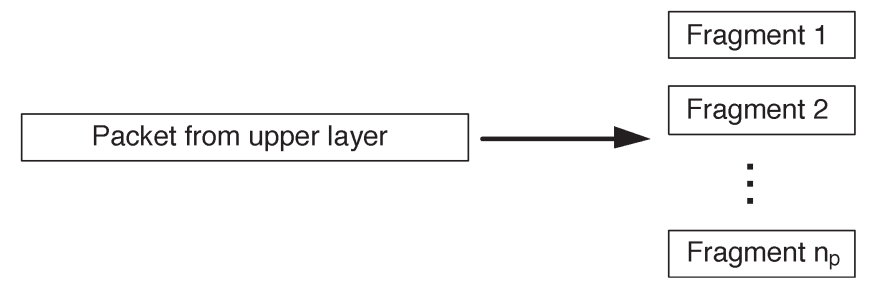

Fig. 11. Packet fragmentation.

for the NCSW ARQ scheme and (3), the throughput of the NCSW scheme can be obtained as

$$
\eta_{\mathrm{NCSW}}=\frac{Y}{Y+X}
$$

In the following section, we use (18) to obtain the average delay and the delay jitter of the NCSW ARQ scheme.

\section{B. Delay Analysis}

The transmission of large frames over a wireless channel is not efficient due to the high probability of frame error [18]. As shown in Fig. 11, large data blocks from the upper layers of the communication-protocol stack (i.e., network layer) are usually broken into smaller fragments to avoid retransmissions of large frames in case of transmission errors. This technique is called packet fragmentation and has been adopted by some existing standards, such as the IEEE 802.11 Medium Access Control (MAC) protocol.

Usually, a sender node is allowed to capture the channel to continuously transmit all fragments of a single packet. Even in a contention-based MAC protocol, the contention mechanism is designed to allow a node to transmit all the fragments as a burst before the next contention period begins. For example, in the IEEE 802.11 MAC protocol as illustrated in Fig. 12, the interframe spacing mechanism is designed to allow a sender to send a sequence of fragments without any interruption. We define an ARQ protocol delay as the time required to complete the transmission of all the fragments of a single packet from the network layer. Since we do not deal with any resource allocation problem in this paper, our delay analysis does not consider the queuing delay. Thus, the transmission delay of an ARQ protocol depends on the packet length and the number of retransmission trials. Without loss of generality, we assume that all packets from the network layer have identical length and are fragmented into $n_{\mathrm{p}}$ frames of identical length $T_{\mathrm{f}}$. Thus, the results can be considered as conditional results for a given value of $n_{\mathrm{p}}$. For the case of variable packet length with a known pdf, we can simply compute the expected value of the results. We also include the ACK/NAK handshake time in the frame length. In the best scenario, when there is no frame error, the entire transmission delay will be equal to $n_{\mathrm{p}} T_{\mathrm{f}}$. However, the average transmission delay is expected to be higher than this ideal value due to possible frame errors. Given the two-state Markov model in Fig. 9, the average delay can be given by

$$
t_{\mathrm{p}}=\frac{X+Y}{Y} \cdot T_{\mathrm{f}}
$$

Next, we introduce a Markov chain with an absorbing state in order to obtain the delay jitter for the NCSW scheme. Let $(q, r)$ be the parameters of the two-state Markov model in Fig. 9 (we replace $[X, Y]$ with $[q, r]$ to simplify the notations). The transmission process of a single fragmented packet can be described by a $\left(2 n_{\mathrm{p}}+1\right)$-state Markov chain with an absorbing state, as shown in Fig. 13.

At the beginning, the system is in $\mathrm{S}_{n_{\mathrm{p}}, T}$ where there are $n_{\mathrm{p}}$ fragments in the transmission queue. If the first transmission succeeds, the system moves to state $\mathrm{S}_{\left(n_{\mathrm{p}}-1\right), \mathrm{T}}$; otherwise, it will move to state $S_{\left(n_{\mathrm{p}}-1\right), \mathrm{R}}$, where it retransmits the first fragment. This transition rule applies to all states, until the system moves into the absorbing state $S_{0, T}$, where all fragments of the packet in the transmission queue have been transmitted successfully. In order to make the mathematical manipulations simple, as shown in Fig. 13, we assign integer indices from 0 to $2 n_{\mathrm{p}}$ to the system states. The initial and the absorbing states are given index numbers $2 n_{\mathrm{p}}$ and 0 , respectively. All $\mathrm{S}_{*, \mathrm{R}}$ states are given odd numbers, and the $S_{*, T}$ states are assigned even numbers from 2 to $2 n_{\mathrm{p}}$. The $\left(2 n_{\mathrm{p}}+1\right) \times\left(2 n_{\mathrm{p}}+1\right)$ state transition probability matrix $(\boldsymbol{\Pi})$ is given in $(21)$, where the $(i, j)$ element denoted by $p_{i j}$ is the probability of transition from state $i$ to state $j$, $\bar{r}=1-r$ and $\bar{q}=1-q$.

$$
\boldsymbol{\Pi}=\left[\begin{array}{ccccccccc}
1 & 0 & 0 & 0 & \cdots & 0 & 0 & 0 & 0 \\
r & \bar{r} & 0 & 0 & \cdots & 0 & 0 & 0 & 0 \\
\bar{q} & q & 0 & 0 & \cdots & 0 & 0 & 0 & 0 \\
0 & 0 & r & \bar{r} & \cdots & 0 & 0 & 0 & 0 \\
0 & 0 & \bar{q} & q & \cdots & 0 & 0 & 0 & 0 \\
\vdots & \vdots & \vdots & \vdots & \ddots & \vdots & \vdots & \vdots & \vdots \\
0 & 0 & 0 & 0 & \cdots & 0 & r & \bar{r} & 0 \\
0 & 0 & 0 & 0 & \cdots & 0 & \bar{q} & q & 0
\end{array}\right] .
$$

This matrix has the structure of

$$
\boldsymbol{\Pi}=\left[\begin{array}{l|c}
\mathbf{I} & \mathbf{0} \\
\hline \mathbf{V} & \mathbf{W}
\end{array}\right]
$$

where $\mathbf{I}$ is a $1 \times 1$ identity matrix, $\mathbf{W}$ is a $2 n_{\mathrm{p}} \times 2 n_{\mathrm{p}}$ matrix containing the probabilities of transition among nonabsorbing states, $\mathbf{V}$ is a $2 n_{\mathrm{p}} \times 1$ matrix that represents the probabilities of transition from the nonabsorbing states to the absorbing state $\mathrm{S}_{0, \mathrm{~T}}$, and $\mathbf{0}$ is a $1 \times 2 n_{\mathrm{p}}$ zero matrix.

Let $D_{i}$ be a random variable representing the number of transitions from a nonabsorbing state $i$ to the absorbing state in Fig. 13. We show in the Appendix that the delay jitter of the NCSW scheme can be given as

$$
\sigma_{\mathrm{p}}=T_{\mathrm{f}} \cdot \sqrt{\left(\delta_{2 n_{\mathrm{p}}}^{2}-d_{2 n_{\mathrm{p}}}^{2}\right)}
$$

where $\delta_{2 n_{\mathrm{p}}}^{2} \triangleq \mathrm{E}\left[D_{2 n_{\mathrm{p}}}^{2}\right]$ and $d_{2 n_{\mathrm{p}}} \triangleq \mathrm{E}\left[D_{2 n_{\mathrm{p}}}\right]$.

\section{Simulation Results}

We simulate a single-hop ad hoc network with one pair of sender-receiver nodes and a varying number of neighbor nodes, as shown in Fig. 2. The channels among the nodes 

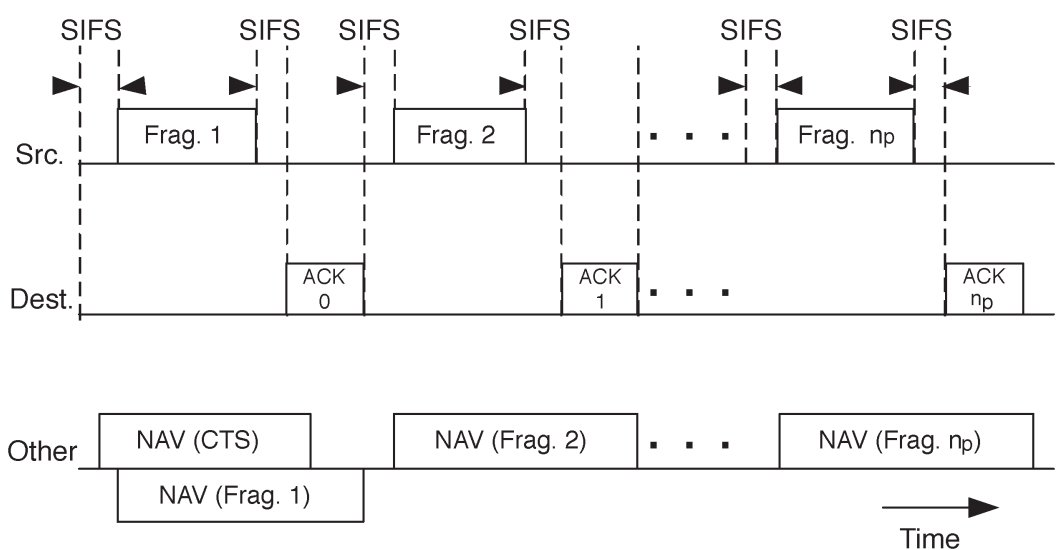

Fig. 12. Transmission of a fragmented packet in the IEEE 802.11 MAC protocol.

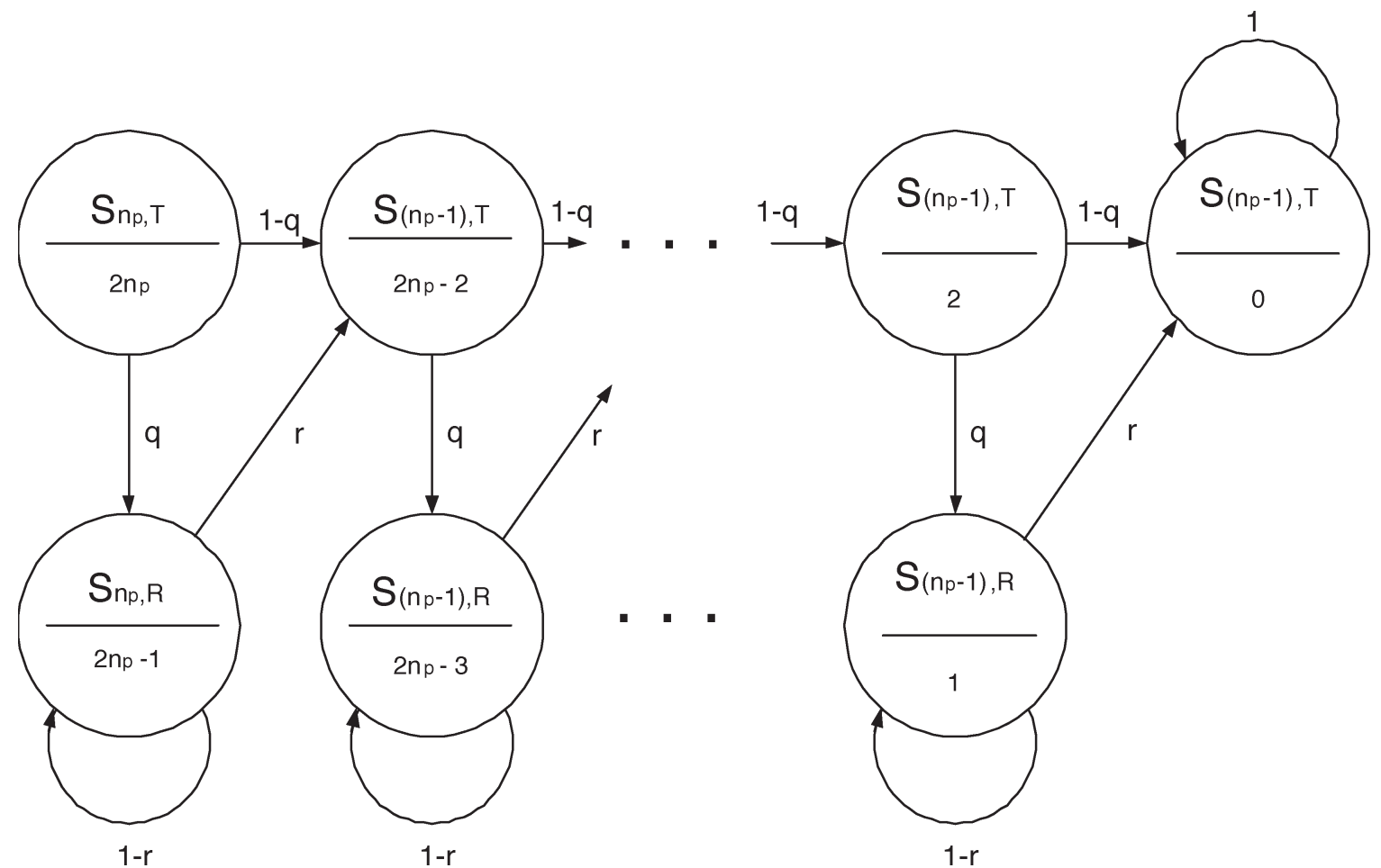

Fig. 13. Markov model for the transmission process of a fragmented packet.

TABLE II

SIMULATION PARAMETERS

\begin{tabular}{lc}
\hline Parameter & Value \\
\hline Carrier frequency & $2400 \mathrm{Mhz}$ \\
Relative speed of mobile nodes & $5 \mathrm{Km} / \mathrm{h}$ \\
Sampling frequency & $8000 \mathrm{samples}$ per second \\
Frame duration & $5 \mathrm{~ms}$ \\
\hline
\end{tabular}

are generated by the Rayleigh fading model. The impacts of path loss and shadowing are not considered due to their very slow variations compared with the activities of a link layer. The Rayleigh fading channels are simulated by low-pass finite impulse response (FIR) filtering of two white Gaussian random processes. The simulation parameters for the fading channels are given in Table II.
The quality of the channel is represented in terms of the ratio of the fading margin over the mean value of the fading envelope as

$$
L=\frac{\sqrt{\gamma}}{\mathrm{E}[|\zeta(t)|]}
$$

The mean value of the fading channels is normalized to a unit; thus, $L=\sqrt{\gamma}$. In other words, variations in the values of the fading margin are translated to the variations in the channel quality. As illustrated in Fig. 14, if the value of the fading margin is increased, the channel quality will be below the threshold value more frequently. This means that as the value of the fading margin is increased, there will be more frame errors, which translates to poorer channel quality. 


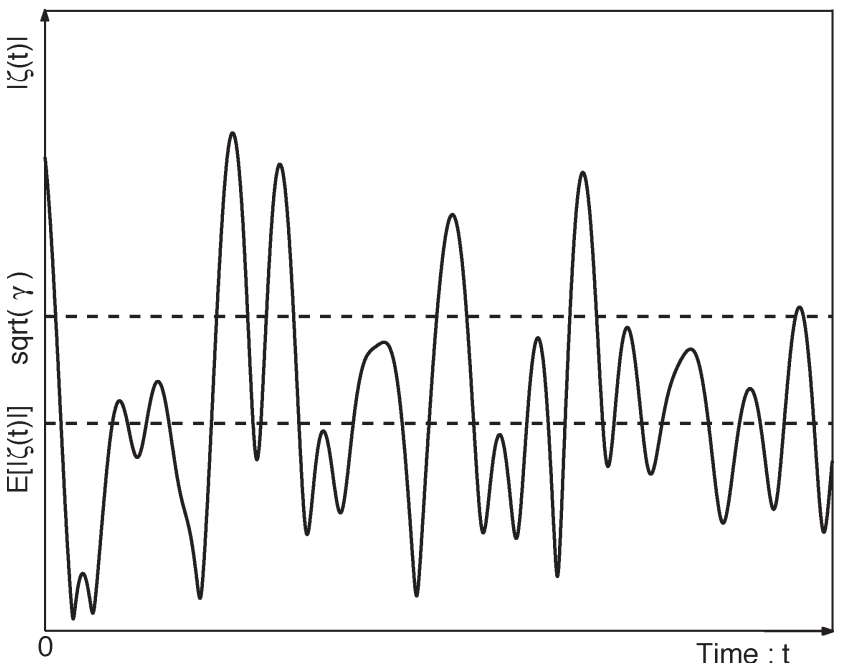

Fig. 14. Concept of a fading margin.

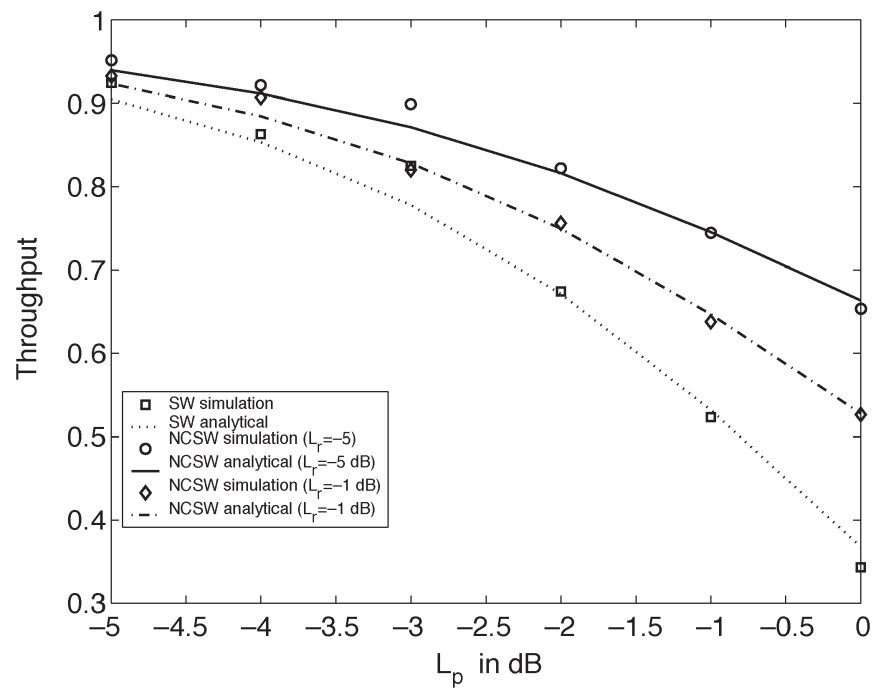

Fig. 15. Throughput versus fading margin of the primary channel.

The data frame duration is set to be $5 \mathrm{~ms}$, which is reasonable for many wireless data networks. A perfect ACK/NAK information on the feedback channels is assumed to be available for the whole cooperation group right after a frame transmission.

In the following sections, we present both the simulation and analytical results for the throughput, the average delay, and the delay jitter of the NCSW scheme.

\section{A. Throughput}

To observe the impact of a small number of cooperative nodes on the system throughput, the SW and the NCSW schemes with only two neighbor nodes are simulated. The throughput values of both schemes are obtained from simulations and analyses. As shown in Fig. 15, the results are plotted against the variations of the quality of the primary channel denoted by $L_{\mathrm{p}}$. The quality of all interim and relay channels are assumed to be identical and denoted by $L_{\mathrm{r}}$. To demonstrate the impact of the variations in the quality of the interim/relay

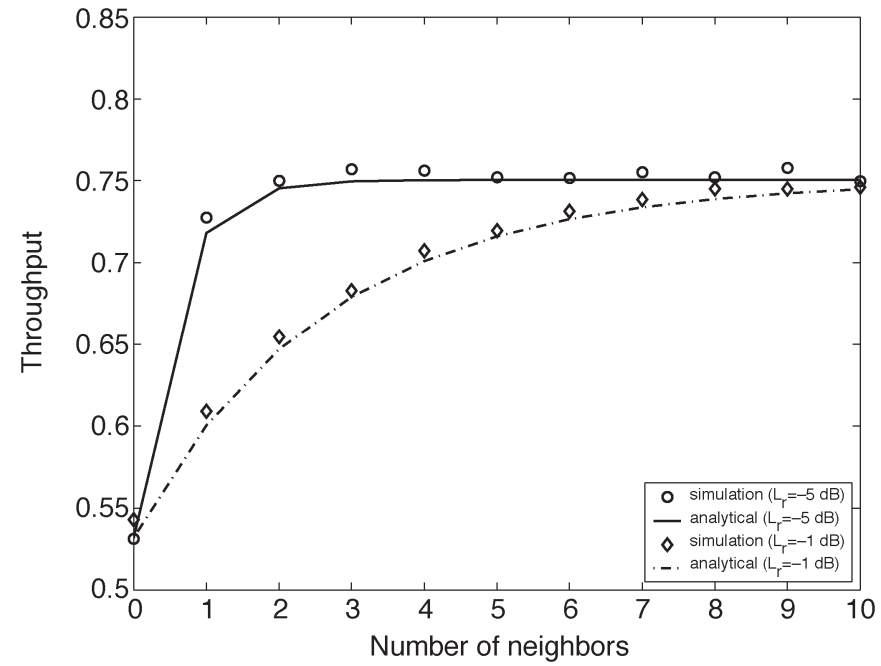

Fig. 16. Throughput versus number of the neighbor nodes $\left(L_{\mathrm{p}}=-1 \mathrm{~dB}\right)$.

channels, the throughput of the NCSW protocol is plotted for two different values of $L_{\mathrm{r}}$, namely, -5 and $-1 \mathrm{~dB}$.

The results in Fig. 15 show that, with cooperation of only two neighbor nodes, the throughput of the NCSW scheme can be improved up to $30 \%$, depending on the quality of the interim/relay channels. A comparison of the analytical and the simulation results also demonstrates the accuracy of the proposed analytical model for the system throughput.

To investigate the impact of the number of neighbor nodes on the protocol throughput, the fading margin of the primary channel is set to $L_{\mathrm{p}}=-1 \mathrm{~dB}$. At this relatively low quality of the primary channel, we can clearly observe the impact of the number of neighbor nodes on the system throughput. Simulations are performed for two different fading margins for the relay/interim channels, namely $L_{\mathrm{r}}=-5 \mathrm{~dB}$ and $L_{\mathrm{r}}=-1 \mathrm{~dB}$. As shown in Fig. 16, when the number of the cooperative nodes increases, the system throughput approaches a saturation level depending on the quality of the primary and the interim/relay channels. If the qualities of the interim/relay channels are good, having only one or two neighbor nodes can significantly improve the system performance; however, when the qualities of the interim/relay channels are poor, more neighbor nodes are required to achieve the same level of performance gain. Saturation of the system throughput is also expected. In fact, regardless of the number of neighbor nodes or their channel qualities, individual frame errors cannot be avoided. However, cooperation of the neighbor nodes can reduce the duration of error bursts.

Another important issue is to separately investigate the impacts of variations in the quality of the interim and the relay channels. The results of this analysis can be helpful in deciding when a node should cooperate. Such a decision can be based on the average quality of the channels from the sender and to the receiver nodes. To keep the system setup consistent with the previous simulations, we obtain the system throughput for only 2 neighbor nodes. We set the fading margin of the primary channel at a fixed level of $L_{\mathrm{p}}=-1 \mathrm{~dB}$ and perform the simulations twice. For the first run, we set the fading margin of the relay channels $L_{\text {relay }}$ at a fixed level of $-5 \mathrm{~dB}$ and let 


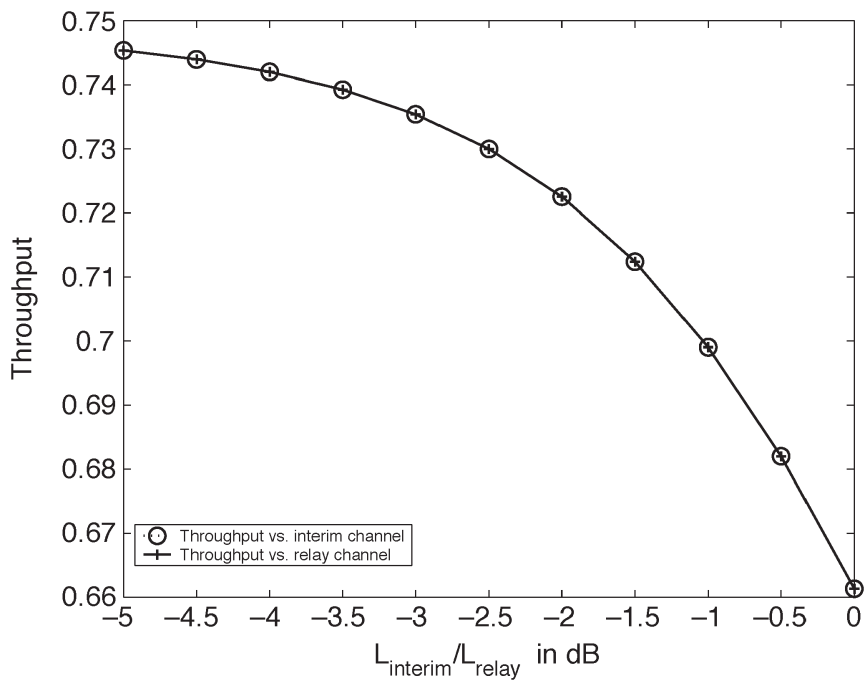

Fig. 17. Throughput versus the fading margin of the interim/relay channels $\left(L_{\mathrm{p}}=-1 \mathrm{~dB}\right)$.

the fading margin of the interim channels $L_{\text {interim }}$ vary from -5 to $0 \mathrm{~dB}$. In the second run, for a fixed fading margin of the interim channels at $-5 \mathrm{~dB}$, we let the fading margin of the relay channels vary from -5 to $0 \mathrm{~dB}$. As shown in Fig. 17, the system throughput follows the same form of variations against the quality of the relay and the interim channels.

\section{B. Average Delay and Delay Jitter}

We simulate the SW and the NCSW protocols with only two cooperating neighbor nodes for our delay analysis. For the NCSW scheme, we assume moderate interim and relay channel qualities at a fading margin of $-2.5 \mathrm{~dB}$. The other channel parameters are the same as in Table II. The fading margin for the primary channel is varied from $-5 \mathrm{~dB}$ (good channel quality) to $0 \mathrm{~dB}$ (bad channel quality). Packets from the upper layer, i.e., network layer, are fragmented into 20 frames of 5-ms durations (including $\mathrm{ACK} / \mathrm{NAK}$ ); therefore, if there is no transmission error, it will take $100 \mathrm{~ms}$ for all the fragments of a single packet to be transmitted. However, as shown in Fig. 18, on the average, it will require more than $100 \mathrm{~ms}$ due to frame errors. As shown in the figure, for a small number of cooperative nodes with moderate interim/relay channel qualities, the NCSW protocol significantly outperforms the SW protocol in terms of transmission delay. For example, when the primary channel is poor $\left(L_{\mathrm{p}}=0 \mathrm{~dB}\right)$, the average delay for the SW scheme is about $265 \mathrm{~ms}$; however, for the same channel condition, the NCSW protocol can reduce the average delay to $160 \mathrm{~ms}$. Thus, due to node cooperation, the average delay is reduced by $60 \%$ when the primary link is experiencing a poor condition.

With the same simulation setup, we also investigate the delay jitter caused by the transmission errors in both schemes. Again, by comparing the results given in Fig. 19, we observe a significant improvement in the performance in terms of reduced delay jitter. For example, when the primary channel is in a poor condition, the delay jitter is reduced from 140 to $20 \mathrm{~ms}$, which is equivalent to an $85 \%$ reduction. Furthermore, the exponential growth in the delay jitter is reduced to a slow linear growth. The

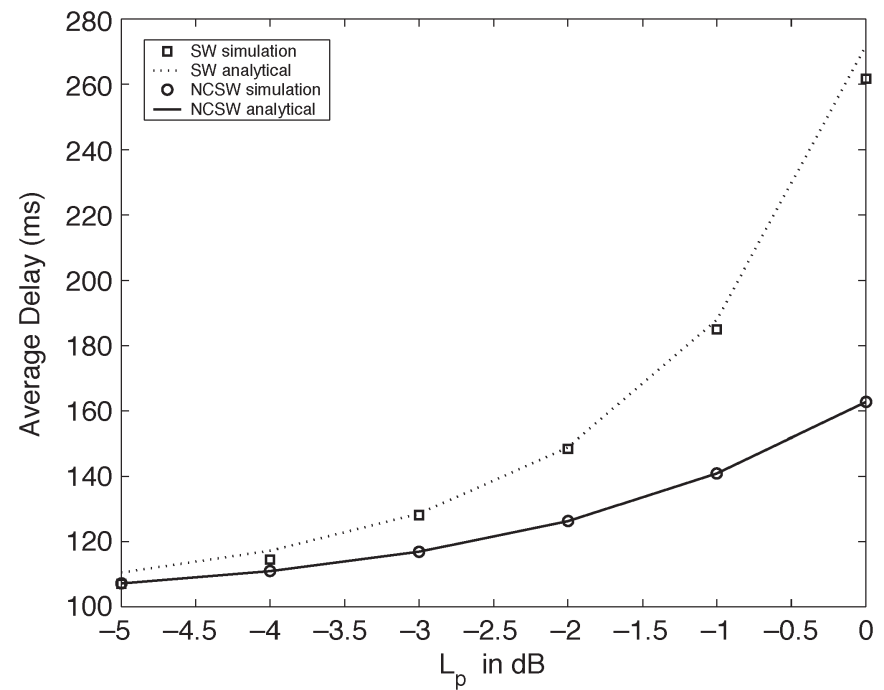

Fig. 18. Delay versus the fading margin of the primary channel.

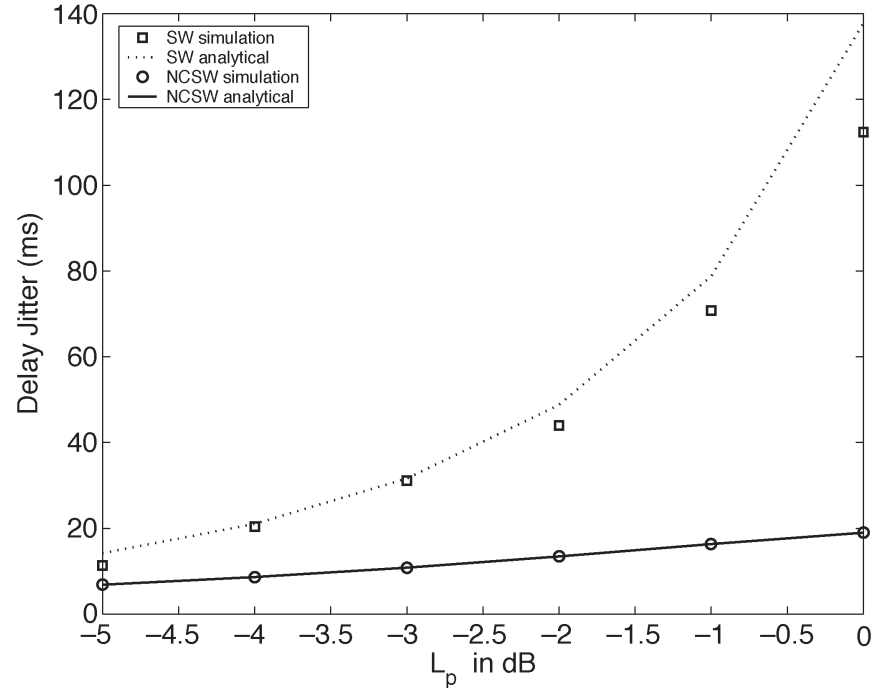

Fig. 19. Jitter versus the fading margin of the primary channel.

improvement of the delay jitter is more remarkable than that of the delay and the throughput. Intuitively, since node cooperation reduces the negative impact of long and varying error bursts, this significant improvement in the jitter is expected. This is a very important advantage of the proposed scheme for real-time multimedia applications, such as voice over Internet protocol (IP), where reducing the delay jitter is a challenging task in providing the desired quality of service.

\section{Conclusion}

We have proposed an NCSW ARQ scheme for wireless ad hoc networks. We developed analytical models for the retransmission process and its tangible performance metrics, such as throughput, average delay, and delay jitter. Simulation results were also given to demonstrate the performance gain and to verify the analytical models. It is concluded that node cooperation can significantly improve the performance of ARQ protocols in wireless ad hoc networks. For example, when 
approximately $50 \%$ of the frames become erroneous due to poor channel condition between the sender and the receiver nodes, the throughput can be improved by up to $30 \%$ with only two cooperating nodes with moderate channel qualities. Meanwhile, the average transmission delay can be reduced by $60 \%$, and the average-delay jitter can be decreased by $85 \%$. The remarkable gains in the average-delay and delayjitter performance are of significant importance for real-time multimedia applications.

Our future research includes the study of the impact of different coding and signal processing techniques at the physical layer and integration issues with the existing MAC protocols, such as the standard IEEE 802.11 MAC protocol.

\section{APPENDIX \\ DERIVATION OF DELAY JITTER}

Delay jitter is the standard deviation of the packet transmission delay, which is defined as

$$
\sigma_{\mathrm{p}} \triangleq \sqrt{\mathrm{E}\left[\left(T_{\mathrm{p}}-t_{\mathrm{p}}\right)^{2}\right]}
$$

We can rewrite (24) as

$$
\sigma_{\mathrm{p}}^{2}=\mathrm{E}\left[\left(D_{\mathrm{p}}-d_{\mathrm{p}}\right)^{2}\right] \cdot T_{\mathrm{f}}^{2}
$$

Then

$$
\sigma_{\mathrm{p}}^{2}=\mathrm{E}\left[\left(D_{2 n_{\mathrm{p}}}-d_{2 n_{\mathrm{p}}}\right)^{2}\right] \cdot T_{\mathrm{f}}^{2}
$$

Since

$$
\mathrm{E}\left[\left(D_{i}-d_{i}\right)^{2}\right]=\mathrm{E}\left[D_{i}^{2}\right]-d_{i}^{2}
$$

we need to obtain $\delta_{i}^{2}=\mathrm{E}\left[D_{i}^{2}\right]$.

Let $d_{j}$ denote the mean value of $D_{j}$ as the average number of steps required to move from a nonabsorbing state $j$ to the absorbing state in Fig. 13. Starting from a nonabsorbing state $j$, the system either moves to the absorbing state in one step or gets to the absorbing state via one or more nonabsorbing states. The probability of the first event is

$\mathbf{P}\{$ go to the absorbing state in one step

$$
\text { |current state is } j\}=p_{j 0} \text {. }
$$

The probability of the second event is

$$
\mathbf{P}\{\text { go to a nonabsorbing state } \mid \text { current state is } j\}=\sum_{i=1}^{2 n_{\mathrm{p}}} p_{j i} \text {. }
$$

If the latter case happens, the time of absorption is $\left(1+D_{i}\right)$. Thus, we have

$$
D_{j}=1 \times p_{j 0}+\sum_{i=1}^{2 n_{\mathrm{p}}} p_{j i}\left(1+D_{i}\right)
$$

and $\delta_{i}^{2}$ is given by

$$
\begin{aligned}
\delta_{i}^{2} & =p_{j 0}+\sum_{i=1}^{2 n_{\mathrm{p}}} \mathrm{E}\left[\left(1+D_{i}\right)^{2}\right] p_{j i} \\
& =p_{j 0}+\sum_{i=1}^{2 n_{\mathrm{p}}}\left(1+2 d_{i}+\delta_{i}^{2}\right) p_{j i} \\
& =\underbrace{p_{j 0}+\sum_{i=1}^{2 n_{\mathrm{p}}} p_{j i}}_{\sum_{i=0}^{2 n_{\mathrm{p}}} p_{j i}=1}+2 \sum_{i=1}^{2 n_{\mathrm{p}}} d_{i} p_{j i}+\sum_{i=1}^{2 n_{\mathrm{p}}} \delta_{i}^{2} p_{j i} \\
& =1+\sum_{i=1}^{2 n_{\mathrm{p}}} d_{i} p_{j i}+\sum_{i=1}^{2 n_{\mathrm{p}}} \delta_{i}^{2} p_{j i} .
\end{aligned}
$$

Organizing (27) into a matrix form, we have

$\left[\begin{array}{c}\delta_{1}^{2} \\ \delta_{2}^{2} \\ \vdots \\ \delta_{\left(2 n_{\mathrm{p}}-1\right)}^{2} \\ \delta_{2 n_{\mathrm{p}}}^{2}\end{array}\right]=\left[\begin{array}{c}1 \\ 1 \\ \vdots \\ 1 \\ 1\end{array}\right]+2 \mathbf{W}\left[\begin{array}{c}d_{1} \\ d_{2} \\ \vdots \\ d_{\left(2 n_{\mathrm{p}}-1\right)} \\ d_{2 n_{\mathrm{p}}}\end{array}\right]+\mathbf{W}\left[\begin{array}{c}\delta_{1}^{2} \\ \delta_{2}^{2} \\ \vdots \\ \delta_{\left(2 n_{\mathrm{p}}-1\right)}^{2} \\ \delta_{2 n_{\mathrm{p}}}^{2}\end{array}\right]$

$\left[\begin{array}{c}\delta_{1}^{2} \\ \delta_{2}^{2} \\ \vdots \\ \delta_{\left(2 n_{\mathrm{p}}-1\right)}^{2} \\ \delta_{2 n_{\mathrm{p}}}^{2}\end{array}\right]=(\mathbf{I}-\mathbf{W})^{-1}\left[\begin{array}{c}1 \\ 1 \\ \vdots \\ 1 \\ 1\end{array}\right]+2(\mathbf{I}-\mathbf{W})^{-1} \mathbf{W}\left[\begin{array}{c}d_{1} \\ d_{2} \\ \vdots \\ d_{\left(2 n_{\mathrm{p}}-1\right)} \\ d_{2 n_{\mathrm{p}}}\end{array}\right]$

Combining (25), (26), and (29), $\sigma_{\mathrm{p}}^{2}$ can be obtained as

$$
\sigma_{\mathrm{p}}=T_{\mathrm{f}} \cdot \sqrt{\left(\delta_{2 n_{\mathrm{p}}}^{2}-d_{2 n_{\mathrm{p}}}^{2}\right)} .
$$

\section{ACKNOWLEDGMENT}

The authors would like to thank the reviewers for the valuable suggestion to simplify the derivation of the average delay in (20).

\section{REFERENCES}

[1] E. V. D. Meulen, "Three-terminal communication channels," Adv. Appl. Probab., vol. 3, pp. 120-154, 1971.

[2] O. Gurbuz and E. Ayanoglu, "A transparent ARQ scheme for broadband wireless access," in Proc. IEEE Wireless Communications and Networking Conf., Atlanta, GA, Mar. 2004, vol. 1, pp. 423-429.

[3] C. Min and W. Gang, "Multi-stages hybrid ARQ with conditional frame skipping and reference frame selecting scheme for real-time video transport over wireless LAN," IEEE Trans. Consum. Electron., vol. 50, no. 1, pp. 158-167, Feb. 2004.

[4] J. Winters, "On capacity of radio communication systems with diversity in a Rayleigh fading environment," IEEE J. Sel. Areas Commun., vol. SAC-5, no. 5, pp. 871-878, Jun. 1987.

[5] I. E. Telatar, "Capacity of multi-antenna Gaussian channels," Eur. Trans. Telecommun., vol. 10, no. 6, pp. 585-595, Nov. 1999. 
[6] V. Tarokh, N. Seshadri, and A. R. Calderbank, "Space-time codes for high data rates wireless communications: Performance criterion and code construction," IEEE Trans. Inf. Theory, vol. 44, no. 2, pp. 744-765, Mar. 1998.

[7] A. Sendonaris, E. Erkip, and B. Aazhang, "Increasing up-link capacity via user cooperation diversity," in Proc. IEEE Int. Symp. Information Theory, Cambridge, MA, Aug. 1998, p. 156.

[8] — , "User cooperation diversity, Part I: System description," IEEE Trans. Commun., vol. 51, no. 11, pp. 1927-1938, Nov. 2003.

[9] —-, "User cooperation diversity, Part II: Implementation aspects and performance analysis," IEEE Trans. Commun., vol. 51, no. 11, pp. 1939-1948, Nov. 2003.

[10] M. Janani, A. Hedayat, T. E. Hunter, and A. Nosratinia, "Coded cooperation in wireless communications: Space-time transmission and iterative decoding," IEEE Trans. Signal Process., vol. 52, no. 2, pp. 362-371, Feb. 2004.

[11] G. L. Stuber, Principles of Mobile Communication. Norwell, MA: Kluwer, 2001.

[12] J. N. Laneman and G. W. Wornell, "Distributed space-time-coded protocols for exploiting cooperative diversity in wireless networks," IEEE Trans. Inf. Theory, vol. 49, no. 10, pp. 2415-2425, Oct. 2003.

[13] H. S. Wang, "On verifying the first-order Markovian assumption for a Rayleigh fading channel model," IEEE Trans. Veh. Technol., vol. 45, no. 2, pp. 353-357, May 1996

[14] M. Zorzi, R. R. Rao, and L. B. Milstein, "ARQ error control for fading mobile radio channels," IEEE Trans. Veh. Technol., vol. 46, no. 2, pp. 445-455, May 1997.

[15] C. Pimentel, T. H. Falk, and L. Lisboa, "Finite-state Markov modeling of correlated Rician-fading channels," IEEE Trans. Veh. Technol., vol. 53, no. 5, pp. 1491-1501, Sep. 2004.

[16] H. Kong and E. Shwedyk, "A hidden Markov model (HMM)-based MAP receiver for Nakagami fading channels," in Proc. IEEE Int. Symp. Information Theory, Whistler, AB, Canada, Sep. 1995, p. 210.

[17] C. H. C. Leung, Y. Kikumoto, and S. A. Sorensen, "The throughput efficiency of the Go-Back-N ARQ scheme under Markov and related error structure," IEEE Trans. Commun., vol. 3, no. 2, pp. 231-233, Feb. 1988.

[18] A. R. Parsad, Y. Shinohara, and K. Seki, "Performance of hybrid ARQ for IP packet transmission on fading channel," IEEE Trans. Veh. Technol., vol. 48, no. 3, pp. 900-910, May 1999.

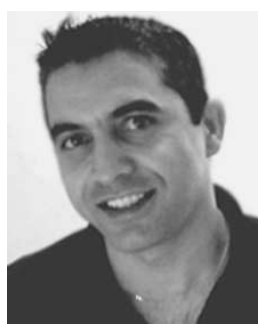

Mehrdad Dianati received the B.S. degree from Sharif University of Technology, Tehran, Iran, in 1992 and the M.S. degree from Khajeh Nasir (K.N.) Toosi University of Technology, Tehran, in 1995, respectively, all in electrical engineering. $\mathrm{He}$ is currently pursuing the Ph.D. degree with the Department of Electrical and Computer Engineering, University of Waterloo, Waterloo, ON, Canada.

His current research interests are performance analysis of wireless network protocols and secure software architectures for loosely connected wireless networks.

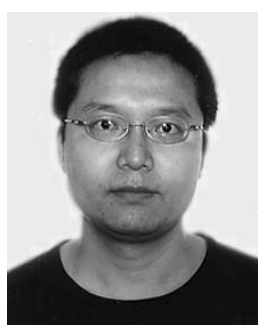

Xinhua Ling received the B.Eng. degree in radio engineering from Southeast University, Nanjing, China, in 1993 and the M.Eng. degree in electrical engineering from the National University of Singapore, Singapore, in 2001 . He is currently pursuing the Ph.D. degree with the Department of Electrical and Computer Engineering, University of Waterloo, Waterloo, ON, Canada.

From July 1993 to May 1998, he was a System Engineer at Beijing Institute of Radio Measurement, Beijing, China. From January 2001 to September 2002, he was a Senior Software Engineer at the Center for Wireless Communications (renamed the Institute of Information Research, Singapore). His research interests are in the area of wireless LAN (WLAN) and ad hoc networks, including Medium Access Control (MAC) protocol design and analysis and cross-layer design for quality-of-service support.

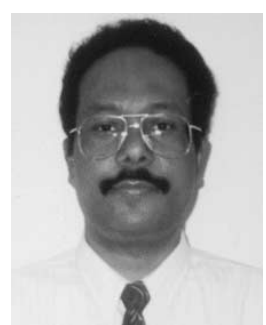

Kshirasagar Naik (M'94) received the B.S. degree from Sambalpur University, Sambalpur, India, in 1981, the M.Tech. degree from the Indian Institute of Technology, Kharagpur, India, in 1983, the M.Math. degree in computer science from the University of Waterloo, Waterloo, ON, Canada, in 1988, and the $\mathrm{Ph} . \mathrm{D}$. degree in electrical and computer engineering from Concordia University, Montreal, QC, Canada, in 1992.

He was a Faculty Member of the University of Aizu, Aizuwakamatsu, Japan, and Carleton University, Ottawa, ON. From May to November 2003, he was a Visiting Associate Professor at the Research Institute of Electrical Communications, Tohoku University, Sendai, Japan. He is currently an Associate Professor in the Department of Electrical and Computer Engineering, University of Waterloo. His research interests include testing of communication protocols, wireless communication, resource allocation in cellular networks, sensor networks, ad hoc networks, Medium Access Control (MAC) protocols, personal area networks, mobile computing, and peer-to-peer communication.

Dr. Naik served as a Program Cochair of the Fifth International Conference on Information Technology held in Bhubaneswar, India, in December 2002. He was a Coguest Editor of a special issue of the IEEE JOURNAL ON SELECTED AREAS IN COMMUNICATIONS (JSAC) on Mobile Computing and Networking published in June 2005. He is currently a Coguest Editor of a special issue of the IEEE Journal on SElEcted AREAS In CommuniCATIONS (JSAC) on Peer-to-Peer Communications and Applications.

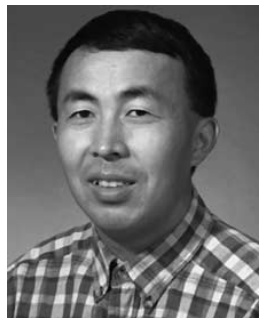

Xuemin (Sherman) Shen (M'97-SM'02) received the B.Sc. degree from Dalian Maritime University, Dalian, China, in 1982 and the M.Sc. and Ph.D. degrees from Rutgers University, Piscataway, NJ, in 1987 and 1990, respectively, all in electrical engineering.

From September 1990 to September 1993, he was first with the Howard University, Washington, DC, and then with the University of Alberta, Edmonton, AB, Canada. Since October 1993, he has been with the Department of Electrical and Computer Engineering, University of Waterloo, Waterloo, ON, Canada, where he is a Professor and the Associate Chair for Graduate Studies. He is the author or coauthor of two books and more than 200 papers and book chapters in wireless communication and network control and filtering. His research interests focus on the mobility and resource management in interconnected wireless/wireline networks, ultra-wideband (UWB) wireless communications systems, wireless security, and ad hoc and sensor networks.

Dr. Shen serves as the Technical Program Committee Chair for Qshine'05, Co-Chair for IEEE Broadnet'05, WirelessCom'05, International Federation for Information Processing (IFIP) Networking'05, 2004 International Symposium on Parallel Architectures, Algorithms and Networks, and IEEE Globecom'03 Symposium on Next Generation Networks and Internet. $\mathrm{He}$ is also an Associate Editor of the IEEE TRANSACTIONS ON Wireless COMMUNiCATIONS, the IEEE TRANSACTIONS ON VehiculaR TeChnology, the Association for Computing Machinery (ACM) Wireless Network, Computer Networks, Dynamics of Continuous, Discrete and Impulsive-Series B: Applications and Algorithms, Wireless Communications and Mobile Computing (Wiley); and Computer Networks (Elsevier). He has been Guest Editor of the IEEE JOURNAL ON SELECTED AREAS IN COMMUNICATIONS (JSAC), IEEE Wireless Communications, and IEEE Communications Magazine. He received the Premier's Research Excellence Award (PREA) from the province of Ontario, Canada, for the demonstrated excellence of his scientific and academic contributions in 2003 and the Distinguished Performance Award from the Faculty of Engineering, University of Waterloo, for his outstanding contribution in teaching, scholarship, and service in 2002. $\mathrm{He}$ is a registered Professional Engineer in Ontario. 